\title{
The improvement of 20" MCP-PMT for neutrino detection
}

\section{Yao Zhu, Zhile Wang}

Harbin Institute of Technology, 150001 Harbin, China

\author{
Shulin Liu, Sen Qian*† \\ Institute of High Energy Physics, Chinese Academy of Sciences, 100049 Beijing, China \\ E-mail: qians@ihep.ac.cn
}

Yiqi Cao, Guorui Huang, Muchun Jin, Zhen Jin, Dong Li, Kun Li, Ling Ren, Shuguang Si, Jianning Sun, Xingchao Wang, Fei Xie, Haoda Zhang

Nanjing, North Night Vision Tech. Ltd., 211106 NanJing, China

\section{Feng Gao}

Physikalisches Institut B, RWTH Aachen university, 52074 Aachen, Germany

\begin{abstract}
For the next generation neutrino experiment, the MCP-PMT collaboration group has done a lot of work in China. After years of hard work, we have developed 20" MCP-PMT. The MCP-PMT has large sensitive area, high quantum efficiency, high gain and large P/V value for good photon counting performance. The high detection efficiency of 20" MCP-PMT will be used in neutrino detection, and we have got an order for $75 \%$ of the PMTs required for neutrino detection. To this end, the MCP-PMT collaboration group also completed the mass production line and batch test system in Nanjing. This presentation will introduce the improvement of 20" MCP-PMT for neutrino detection.
\end{abstract}

ICHEP 2018, International Conference on High Energy Physics

4-11 July 2018

Seoul, Korea

${ }^{*}$ Speaker.

${ }^{\dagger}$ On behalf of the MCP-PMT workgroup. 


\section{Introduction}

In 2013, the 8 inch MCP-PMT was produced with two shapes, vertical and horizontal ones, both of these two types of MCP-PMTs have the best MCP modules for the single photon detection, and the P/V of the SPE of them is better than the Dynode-PMTs. In 2014, the 20 inch glass was produced, and also the MCP-PMT was produced with two shapes, vertical and horizontal ones, and both of the two shapes were good in the SPE test. The performances of these types of 20 inch MCP-PMT were as good as the 8 inch ones. In 2015, JUNO (Jiangmen Underground Neutrino Observatory) ordered 15000 pieces 20" MCP-PMT from the NNVT [1-4].

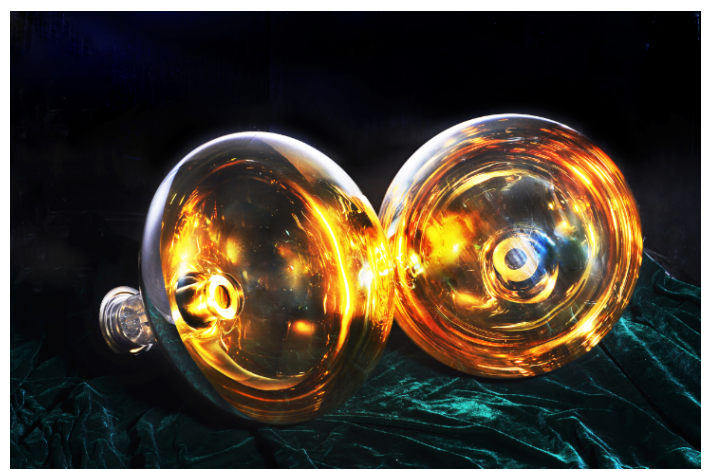

Figure 1: 20" MCP-PMT

The mass production line was builted by MCP-PMT collaboration group in 2016. At present, about 30 PMTs are produced every day. According to the requirement of JUNO project, each production PMT must be carefully tested. The batch test system has been designed and finished at the beginning of 2017. All the PMTs are tested in the factory before the delivery time. So far, we have tested about 7000 MCP-PMTs. As illustrated in Table 1, the parameters of the photocathodes are really improved during the mass production process. It is worth noting that the time characteristics of MCP-PMTs is not good enough. That is due to changes in the collection, the photocathode and technics of MCP.

Table 1: MCP-PMT performance parameters in the mass production by batch test

\begin{tabular}{|c|c|c|c|}
\hline PMT parameters & Data in the contract & MCP-PMT prototype & $\sim 7000$ PMTs \\
\hline QE@400nm(\%) & $\geq 26.5$ & $\sim 26$ & 30.1 \\
\hline QE Uniformity(\%) & $\leq 15$ & $\leq 10$ & 7.3 \\
\hline P/V of SPE & $\geq 2.8$ & $\sim 5.6$ & 7.08 \\
\hline HV@ $10^{7}(\mathrm{~V})$ & 2800 & $\sim 1930$ & 1745 \\
\hline DE@400nm(\%) & - & $\sim 26$ & 30.1 \\
\hline DE@420nm(\%) & $\geq 24$ & - & 27.61 \\
\hline DR(KHz) & $\leq 50$ & $\sim 30$ & 37.25 \\
\hline TTS(ns) & $\leq 15$ & $\sim 12$ & 20 \\
\hline APR $(\%)$ & $\leq 5$ & $\leq 2.5$ & 0.63 \\
\hline Linearity $<10 \%(\mathrm{pe})$ & $\geq 1000$ & $\sim 1000$ & 1299 \\
\hline RT(ns) & $\leq 2$ & $\sim 1.2$ & 1.41 \\
\hline FT(ns) & $\leq 12$ & $\sim 10.2$ & 24.91 \\
\hline
\end{tabular}




\section{The improvement of $\mathrm{CE}$}

At first we did lots of work to improve the QE and CE, but the CE was only 70\%, not the same as we designed. The MCP-PMT work group did the best to improve the CE, such as using some special technology about the MCPs, adjusting the depth of output electrode, changing the diameter of MCP and channels, modifying the bias angle of channel, and so on. Finally, the CE of the MCP-PMTs was improved from $70 \%$ to $100 \%$ successfully [5]. As shown in Table 2, we tested 30 samples of Dynode-PMTs and MCP-PMTs, respectively. The results show that the collection efficiency of MCP-PMT is about $100 \%$, which is better than Dynode-PMT's.

Table 2: Performance comparison of Dynode-PMT and MCP-PMT (DE=QE*CE)

\begin{tabular}{|c|c|c|c|}
\hline 30 pics 20" PMT & DE@ 420nm $(\%)$ & QE@ 420nm $(\%)$ & CE@ 420nm $(\%)$ \\
\hline Dynode-PMT & $28.08 \pm 1.02$ & $30.48 \pm 0.87$ & $93.19 \pm 1.44$ \\
\hline MCP-PMT & $27.15 \pm 0.32$ & $27.56 \pm 0.4$ & $101.4 \pm 1.2$ \\
\hline
\end{tabular}

\section{The improvement of $\mathrm{QE}$}

What's more, we have adopted many methods to improve QE. Firstly, we use a method of evaporating metal film on the cathode substrate to increase the electron supplement ability of photocathode. Secondly, a transmissive film is vapor deposited on the substrate for enhancing the transmittance of incident light. Thirdly, we improve the cathode fabrication process and optimized different material compositions to improve photocathode performance as much as possible. In addition, we strictly controll the processing of the glass bulb to make the surface clean.

Therefore, the quantum efficiency of the photocathode is really improved during the mass production process. As shown in Figure 2(a), the QE of the photocathode is improved from $30 \%$ to $35 \%$ at the peak wavelength. These MCP-PMTs have high QE and we call them HQE-MCPPMTs. At present, the QE of MCP-PMT is better than that of the Dynode-PMT. So far, we have tested about 7000 MCP-PMTs. As can be seen from Figure 2(b), the QE of MCP-PMT is steadily improved. In particular, the latest two batches about 500 PMTs are HQE-PMTs.

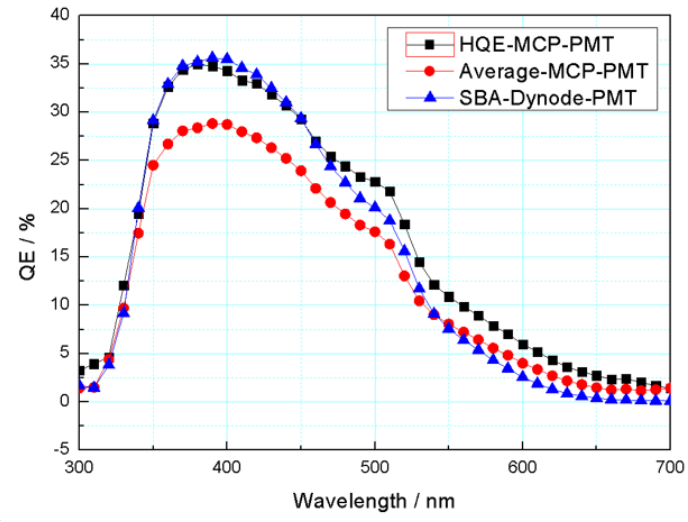

(a) the photocathode spectral response

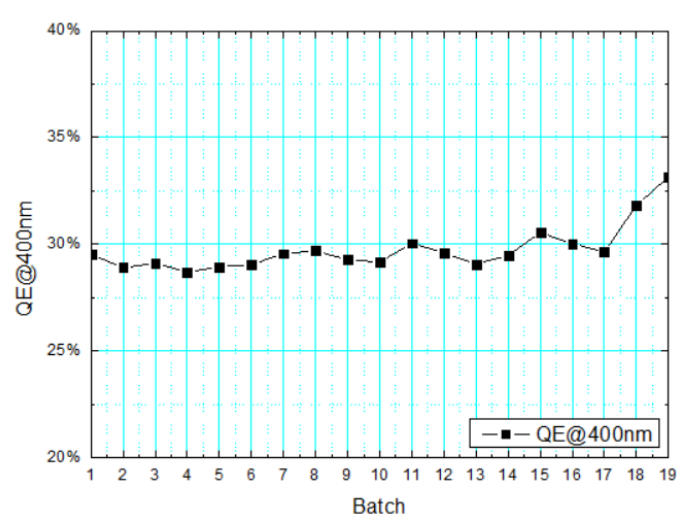

(b) the average QE with different batch

Figure 2: The improvement of quamtum efficiency 
Accordingly, the DE of MCP-PMT is also improved by the increasing of CE and QE. For the latest batch test of 336 HQE-MCP-PMTs, as shown in Figure 3, the average DE of these PMTs is $30.32 \%$ at $420 \mathrm{~nm}$.

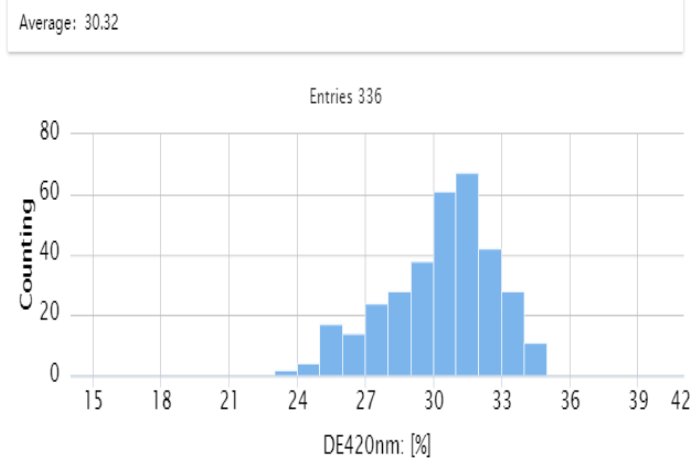

Figure 3: The DE of HQE-MCP-PMTs

\section{Conclusions}

The MCP-PMT group has already produced 7000 MCP-PMTs for JUNO. The average ED of these MCP-PMTs is $27.61 \%$ at $420 \mathrm{~nm}$. What's more, we can produce HQE-MCP-PMTs with some technical improvements. The HQE-MCP-PMTs, whose DE is more than $30 \%$ at $420 \mathrm{~nm}$, will be used on JUNO project.

\section{Acknowledgments}

The MCP-PMT development project has been partially supported by the Strategic Priority Research Program of the Chinese Academy of Sciences (Grant No. XDA10010400) and the National Natural Science Foundation of China (Grant No.11175198 and No.11475209 and No.11675205 and No.11675196).

\section{References}

[1] Y. Chang, G. Huang, Y. Heng, et al., The R\&D of the 20" MCP-PMTs for JUNO, Nuclear Inst \& Methods in Physics Research A, 2016, 824:143-144.

[2] S. Liu and B. Yan, Development of the large area MCP-PMT, Proceedings of SPIE - The International Society for Optical Engineering, 2014, 9284:928402-928402-10.

[3] F. Gao, G. Huang, Y. Heng, et al., Status of the 20 inch MCP-PMT prototype development for JUNO experiment, 2017:012050.

[4] J. Xia, S. Qian, W. Wang, et al., A performance evaluation system for photomultiplier tubes, Journal of Instrumentation, 2015, 10(3):P03023-P03023.

[5] L. Chen, J. Tian, C. Liu, et al., Optimization of the electron collection efficiency of a large area MCP-PMT for the JUNO experiment, Nuclear Inst \& Methods in Physics Research A, 2016, 827:124-130. 